\title{
Levantamento e avaliação da incidência das doenças da mandioca no estado da Paraíba
}

\author{
Martival dos Santos Morais ${ }^{1 *}$, Luciana Cordeiro do Nascimento ${ }^{2}$, Keila Aparecida Moreira ${ }^{3}$, Marilene da Silva \\ Cavalcanti $^{1}$, Neiva Tinti de Oliveira ${ }^{1}$
}

${ }^{1}$ Universidade Federal de Pernambuco, UFPE, Departamento de micologia, R. Prof. Nelson Chaves s/n, Cidade Universitária, CEP 50670-420 Recife, PE. ${ }^{2}$ Universidade Federal da Paraíba, UFPB, Departamento de Fitossanidade, CEP 58397-000, Cx postal 04, Areia, PB. ${ }^{3}$ Universidade Federal Rural de Pernambuco, UfRPE, Departamento de Morfologia e Fisiologia Animal, Av. Bom Pastor, s/n, Boa Vista,CEP 55296-901 Garanhuns, PE.

Autor para correspondência: Martival dos Santos Morais (martivalcg@hotmail.com)

Data de chegada: 01/04/2013. Aceito para publicação em: 28/06/2013.

\section{RESUMO}

Morais, M.S.; Nascimento, L.C.; Moreira, K.A.; Cavalcanti, M.S.; Oliveira, N.T. Levantamento e avaliação da incidência das doenças da mandioca no estado da Paraíba. Summa Phytopathologica, v.39, n.3, p.204-206, 2013.

\begin{abstract}
Objetivando-se levantar e quantificar a incidência de doenças da mandioca em plantios comerciais no Estado da Paraíba, realizaram-se coletas em 21 municípios de quatro microrregiões do Estado no primeiro semestre de 2011. As doenças detectadas foram mancha parda (Cercosporidium henningsii), mancha branca (Phaeoramularia manihotis), antracnose (Colletotrichum gloeosporioides f. sp. manihotis) e podridão mole (Fusarium oxysporum). A incidência apresentou-se com
\end{abstract}

variação, sendo os maiores índices constatados nos municípios das microrregiões do Curimataú Ocidental, Sapé e Brejo paraibano. A mancha parda ocorreu em todos os municípios avaliados, sendo seguida da mancha branca, cuja porcentagem de plantas atacadas foi inferior. A antracnose foi detectada em cerca de $70 \%$ dos municípios avaliados, porém com incidência reduzida. A maior incidência da podridão causada por $F$. oxysporum foi observada na cidade de Imaculada, microrregião de Serra do Teixeira.

Palavras-chave adicionais: Quantificação, etiologia, manchas, podridão, microrregiões.

\section{ABSTRACT}

Morais, M.S.; Nascimento, L.C.; Moreira, K.A.; Cavalcanti, M.S.; Oliveira, N.T. Survey and evaluation of the incidence of cassava diseases in the state of Paraíba. Summa Phytopathologica, v.39, n.3, p.204-206, 2013.

To survey and quantify the incidence of the diseases affecting cassava (Manihot esculenta) in commercial crops of the state of Paraiba - Brazil, samplings were conducted in 21 municipalities at four microregions of the state during the first semester of 2011. The detected diseases were brown leaf spot (Cercosporidium henningsii), white leaf spot (Phaeoramularia manihotis), anthracnose (Colletotrichum gloeosporioides f. sp. manihotis) and root rot (Fusarium oxysporum). The incidence varied, and the highest indexes were found for the municipalities of the microregions of Curimataú Ocidental, Sapé and Brejo Paraibano. Brown leaf spot occurred in all evaluated municipalities, followed by white leaf spot, which showed inferior percentage of affected plants. Anthracnose was detected for around $70 \%$ of the evaluated municipalities but had reduced incidence. The highest incidence of root rot caused by $F$. oxysporum was observed for the city Imaculada, microregion of the Serra do Teixeira.

Additional keywords: Quantification, etiology, spots, rot, microregions.

A mandioca é cultivada em todas as regiões do Brasil, tendo-se como maiores produtoras as regiões Norte e Nordeste, com 32,8 e $29,7 \%$ (4). A maior parte da produção nestas regiões é destinada à indústria artesanal de farinha, enquanto as raízes e a folhagem desta planta são utilizadas como alimento para animais (3). Várias doenças foram registradas causando prejuízos na produtividade desta cultura, como: podridões radiculares, manchas foliares, bacterioses, superalongamento e viroses (6).

No Estado da Paraíba, a mandioca apresenta-se no interior como cultura de subsistência e adquire caráter comercial no litoral, microrregião do Brejo e áreas circunvizinhas (8). Não foram ainda realizados estudos acerca da identificação de possíveis agentes infecciosos fúngicos da cultura no Estado.
O presente trabalho teve então como objetivo, determinar a etiologia das doenças da mandioca em plantios comerciais do Estado da Paraíba e relacionar a incidência com a pluviosidade.

As coletas de dados de campo foram realizadas nos meses de janeiro a junho de 2011 e o levantamento em 21 municípios distribuídos em quatro microrregiões paraibanas (Figura 1). As amostras, constituídas de folhas e raízes sintomáticas, foram enviadas para isolamento e identificação no Laboratório de Fungos Fitopatogênicos da Universidade Federal de Pernambuco. O isolamento dos fungos,foi realizado em placas de Petri contendo meio de cultura BDA e câmara úmida, sendo identificados em microscópio óptico com auxílio de literatura especializada $(2,9)$. Nos testes de patogenicidade foram utilizadas folhas e raízes de mandioca provenientes da cidade de 
Imaculada - PB. As folhas foram inoculadas com uma suspensão de $10^{6}$ conídios $\mathrm{mL}^{-1}$ e fragmentos radiculares com discos de $10 \mathrm{~mm}$ de diâmetro oriundo de culturas de F. oxysporum isolado.

Os dados de precipitação pluviométrica acumulada no mês anterior e meses posteriores das coletas em cada município foram fornecidos pelo Laboratório de Meteorologia da Agência Executiva de Gestão de Águas da Paraíba (Figura 1).

Para análise estatística, as médias da incidência das doenças nos municípios foram comparadas aplicando-se a análise de variância e o teste de Scott-Knott a 5\% de probabilidade. O processamento dos dados foi realizado utilizando-se o programa Software SAS, desenvolvido pelo SAS Institute, ano 1992.

As doenças detectadas nos campos agrícolas do Estado da Paraíba foram mancha parda (Cercosporidium henningsii Allescher); mancha branca [Phaeoramularia manihotis (F. Stevens \& Solheim) MB Ellis]; antracnose, [Colletotrichum gloeosporioides f. sp. manihotis Henn (Penn.)] e a podridão mole (Fusarium oxysporum Schlent). Os maiores valores de incidência de doenças foram constatados nos municípios pertencentes às microrregiões do Brejo Paraibano, seguida do Curimataú Ocidental e Sapé (Tabela 1).

Mancha Parda: ocorreu em todos os municípios (Tabela 1). Outros autores confirmaram também a presença da doença em praticamente todos os locais de cultivo $(5,10)$. Os sintomas desta doença são manchas de 5 a $10 \mathrm{~mm}$ de diâmetro, amareladas a pardo-avermelhadas, de formato angular e contornadas por bordas escuras (6). Os municípios que apresentaram maior número de plantas afetadas pela mancha parda foram Lagoa Grande, Lagoa Nova e Areia, com 93,15\%; 92,75 e 90,10\%, pertencentes à microrregião Brejo paraibano. Houve incremento da doença em consequência do aumento do índice de precipitação do mês anterior à coleta, o que é corroborado por Lozano \& Booth (6) (Figura
1). Nos municípios que apresentaram alta incidência da doença, é possível prever queda de produtividade $(5,10)$.

Mancha Branca: na maioria dos municípios de ocorrência registrouse baixa taxa de incidência da doença. Os municípios em que foram observados os maiores percentuais pertencem às microrregiões Brejo Paraibano e Sapé. A mancha branca é encontrada mais comumente em regiões úmidas e frias (6), correspondendo ao clima da microrregião Brejo Paraibano. Em Riachão do Poço, situado na região ocidental de Sapé, ocorreu a maior incidência, $52 \%$, não havendo relação direta entre o índice da doença e a precipitação. Na microrregião de Sapé, a doença está relacionada à preferência do patógeno a áreas situadas em baixadas e próximas ao litoral (Tabela 1). Os sintomas para mancha branca são lesões circulares ou angulares, medindo de 1-7 mm de diâmetro brancas ou marrons claras (6).

Antracnose: a antracnose causa cancros nas hastes, ramos e frutos, manchas foliares e morte dos ponteiros $(9,10)$. A doença foi encontrada em pouco mais de $70 \%$ dos municípios. A maior incidência ocorreu no Brejo Paraibano, junto aos municípios: Lagoa Nova, Matinhas e Areia com $43,15 \% ; 32,5 \%$ e $20 \%$.

Podridão Radicular mole: os sintomas reflexos manifestados pelo ataque dos patógenos são murcha e queda de folhas, enquanto o sintoma direto é o apodrecimento progressivo dos tecidos radiculares. A maior incidência da podridão mole, causada por $F$. oxysporum foi observada na cidade de Imaculada, na microrregião de Serra do Teixeira, atingindo $21 \%$ das plantas (Tabela 1). Bandyopadhyay et al. (1), também registraram que em Camarões, $30 \%$ das tuberosas apodrecidas foram atacadas por Fusarium, sendo as principais espécies identificadas, $F$. oxysporum e F. solani e no Estado do Pará, Poltronieri et al. (7), relataram a morte de $30 \%$ das mudas de mandioca da variedade Olho de Boto, causada pela ação de $F$. solani.

Tabela 1. Incidências (\%) de plantas de mandioca sintomáticas de doenças no Estado da Paraíba $-1^{\circ}$ semestre de 2011

\begin{tabular}{|c|c|c|c|c|c|c|c|}
\hline \multicolumn{2}{|c|}{ MP } & \multicolumn{2}{|c|}{ MB } & \multicolumn{2}{|c|}{ AC } & \multicolumn{2}{|l|}{ PR } \\
\hline Lagoa Grande & $93,15 \mathrm{a}^{1}$ & Riachãodo poço & $52,00 \mathrm{a}$ & Lagoa nova & $43,15 \mathrm{a}$ & Imaculada & $21,00 \mathrm{a}$ \\
\hline Lagoa Nova & $92,75 \mathrm{a}$ & Sapé & $22,15 b$ & Matinhas & $32,50 \mathrm{a}$ & Princesa Isabel & $11,70 \mathrm{~b}$ \\
\hline Areia & $90,10 \mathrm{a}$ & Lagoa Nova & $19,00 \mathrm{~b}$ & Areia & $20,00 \mathrm{~b}$ & Remígio & $0,00 \mathrm{c}$ \\
\hline S. M. de Taipú & $88,50 \mathrm{a}$ & Areia & $15,00 \mathrm{c}$ & Jurú & $10,00 \mathrm{c}$ & Areia & $0,00 \mathrm{c}$ \\
\hline Cuité & $88,33 \mathrm{a}$ & Remígio & $12,50 \mathrm{c}$ & Teixeira & $7,95 \mathrm{c}$ & Pilões & $0,00 \mathrm{c}$ \\
\hline Lagoa Seca & $82,15 \mathrm{a}$ & Matinhas & $9,70 \mathrm{~d}$ & Sapé & $4,50 \mathrm{c}$ & Tavares & $0,00 \mathrm{c}$ \\
\hline Sapé & $81,50 a$ & Cuité & $5,05 \mathrm{e}$ & Nova Floresta & $3,65 \mathrm{c}$ & Jurú & $0,00 \mathrm{c}$ \\
\hline S. J. dos Ramos & $81,00 \mathrm{a}$ & S. J. dos Ramos & $2,60 \mathrm{e}$ & Pocinhos & $3,15 \mathrm{c}$ & Teixeira & $0,00 \mathrm{c}$ \\
\hline Sobrado & $72,70 \mathrm{a}$ & S. M. de Taipú & $2,35 \mathrm{e}$ & S. M. de Taipú & $2,50 \mathrm{c}$ & Soledade & $0,00 \mathrm{c}$ \\
\hline Pilões & $65,85 \mathrm{a}$ & Imaculada & $1,75 \mathrm{e}$ & Sobrado & $2,00 \mathrm{c}$ & Pocinhos & $0,00 \mathrm{c}$ \\
\hline Matinhas & $56,15 \mathrm{a}$ & Soledade & $1,65 \mathrm{e}$ & Pilões & $1,30 \mathrm{c}$ & Nova Floresta & $0,00 \mathrm{c}$ \\
\hline Imaculada & $46,00 b$ & Lagoa Grande & $1,65 \mathrm{e}$ & Remígio & $1,00 \mathrm{c}$ & Cuité & $0,00 \mathrm{c}$ \\
\hline Teixeira & $44,60 b$ & Princesa Isabel & $1,40 \mathrm{e}$ & Soledade & $0,70 \mathrm{c}$ & Lagoa Seca & $0,00 \mathrm{c}$ \\
\hline Princesa Isabel & $13,90 b$ & Pilões & $1,30 \mathrm{e}$ & Cuité & $0,30 \mathrm{c}$ & Sapé & $0,00 \mathrm{c}$ \\
\hline Remígio & $12,00 \mathrm{~b}$ & Teixeira & $1,30 \mathrm{e}$ & Princesa Isabel & $0,30 \mathrm{c}$ & Sobrado & $0,00 \mathrm{c}$ \\
\hline Jurú & $9,60 \mathrm{~b}$ & Tavares & $1,30 \mathrm{e}$ & Lagoa Seca & $0,00 \mathrm{c}$ & S. M. de Taipú & $0,00 \mathrm{c}$ \\
\hline Soledade & $9,00 \mathrm{~b}$ & Pocinhos & $0,70 \mathrm{e}$ & S. J. dos Ramos & $0,00 \mathrm{c}$ & Riachão do poço & $0,00 \mathrm{c}$ \\
\hline Nova Floresta & $8,95 b$ & Sobrado & $0,00 \mathrm{e}$ & Lagoa Nova & $0,00 \mathrm{c}$ & S. J. dos Ramos & $0,00 \mathrm{c}$ \\
\hline Riachão do poço & $4,20 b$ & Lagoa Seca & $0,00 \mathrm{e}$ & Matinhas & $0,00 \mathrm{c}$ & Lagoa Grande & $0,00 \mathrm{c}$ \\
\hline Pocinhos & $3,00 \mathrm{~b}$ & Jurú & $0,00 \mathrm{e}$ & Areia & $0,00 \mathrm{c}$ & Lagoa Nova & $0,00 \mathrm{c}$ \\
\hline Tavares & $0,72 b$ & Nova Floresta & $0,00 \mathrm{e}$ & Jurú & $0,00 \mathrm{c}$ & Matinhas & $0,00 \mathrm{c}$ \\
\hline
\end{tabular}

${ }^{1}$ Médias de mesmas letras não diferem entre si estatisticamente (Teste de Scott-Knotta 5\% de probabilidade).

${ }^{2}$ Doenças: MP - Mancha parda; MB - Mancha branca; AC - Antracnose; PR - Podridão radicular. 


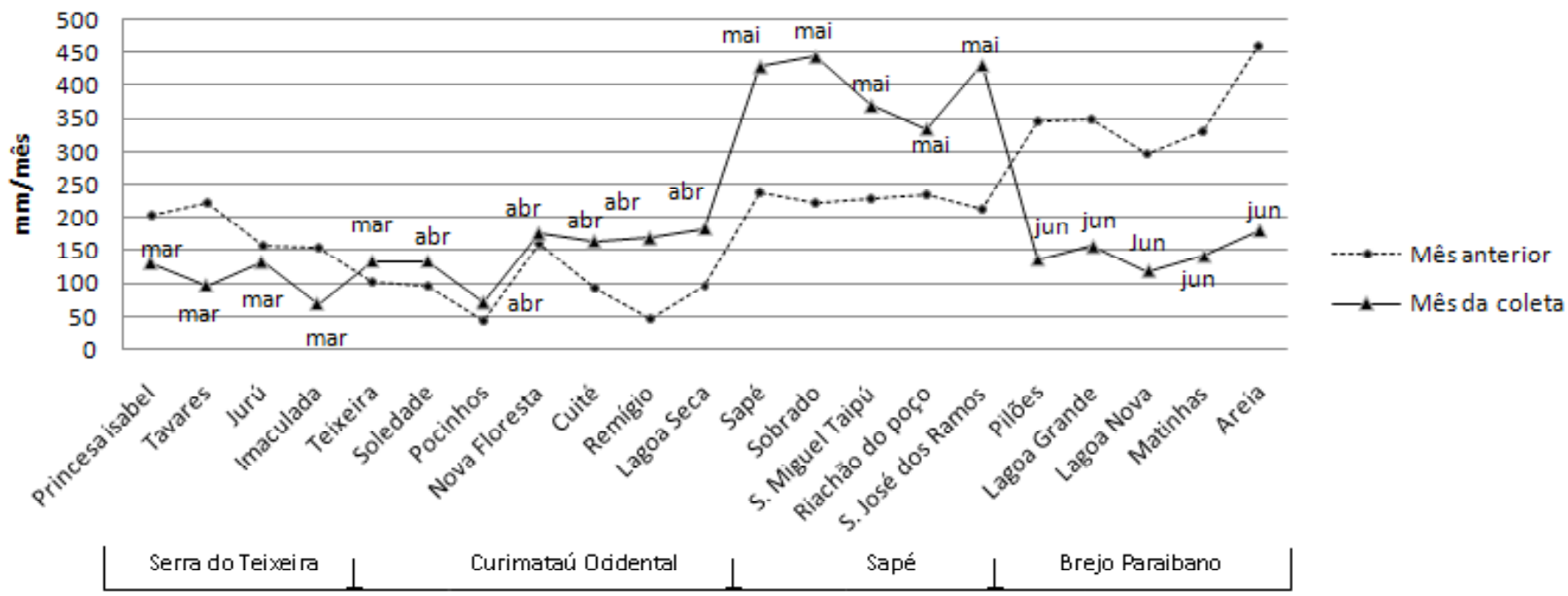

Figura 1. Precipitação bimensal acumulada (mês anterior e exato da coleta) em municipios do Estado da Paraíba - Primeiro semestre de 2011.

\section{AGRADECIMENTOS}

Os autores agradecem ao Programa de Pós-graduação em Biologia de Fungos da Universidade Federal de Pernambuco e à Coordenação de aperfeiçoamento de pessoal de nível superior pela concessão de recursos laboratoriais e financeiros.

\section{REFERENCIAS BIBLIOGRÁFICAS}

1. Bandyopadhyay, R.; Mwangi, M.; Aigbe, S. O.; Leslie, J. F. Fusarium species from the cassava root rot complex in West Africa. Phytopathology, St. Paul, v. 96, p. 673-676, 2006.

2. Chupp, C. A. Monograph of the fungus Genus Cercospora. New York: Ithaca, 1954. $667 \mathrm{p}$.

3. Furlaneto, F. P. B.; Kanthack, R. A. D.; Bonissoni, C . O agronegócio da mandioca na região paulista do médio Paranapanema. Análises e indicadores do agronegócio, São Paulo, v. 1, n. 4, 2006. Disponível em: 〈http://www.iea.sp.gov.br/out/verTexto.php? $\operatorname{codTexto}=5280$ 〉.
Acesso em: 09 set. 2009.

4. Instituto Brasileiro de Geografia e Estatística. Levantamento Sistemático da Produção Agrícola. Rio de Janeiro, 2012. Disponível: <http:// www.ibge.gov.br/home/estatistica/indicadores/agropecuária/Ispa>, Acesso em: 13 ago 2012.

5. Lozano, J. C. Outbreaks of cassava diseases and losses induced. Fitopatologia Brasileira, Brasília, DF, v. 14, p. 7-11, 1989.

6. Lozano, J. C.; Booth, R. H. Diseases of Cassava (Manihot esculenta Crantz). Pest articles and Summaries, Chicago, n. 20, p. 30-54, 1974.

7. Poltronieri, L. S.; Trindade, D. R.; Albuquerque, F. C.; Duarte, M. L. R.; Cardoso, S. S. Incidência de Fusarium solani em mandioca no Estado do Pará. Fitopatologia Brasileira, Brasília, DF, v. 5, p. 544, 2002.

8. Rodriguez, J. L. Atlas da Paraíba: espaço geo-histórico e Cultural. 3. ed. João Pessoa: Grafset, 2002. 112 p.

9. Sutton, B. C. The coelomycetes. Surrey: Commonwealth Mycological Institute, 1980. 696p.

10. Teri, J. M.; Lozano, J.C.; Thurston, H. D. Epidemiology of cassava brown leaf spot. Fitopatologia Brasileira, Brasília-DF, v. 6, n. 3, p. 314-344, 1981 